\title{
Multiple myeloma-associated skin light chain amyloidosis: A case of misdiagnosis
}

\author{
LI XIAO $^{1}$, FENGXIA LIN $^{1}$, RONG XIAO ${ }^{2}$, CHUN HU $^{1}$, MINGYANG DENG $^{3}$, \\ DAIQIANG LI ${ }^{4}$, XIAOLING SHE ${ }^{4}$, FUYOU LIU ${ }^{1}$ and LIN SUN ${ }^{1}$ \\ Departments of ${ }^{1}$ Nephrology, ${ }^{2}$ Dermatology, ${ }^{3}$ Hematology and ${ }^{4}$ Pathology, Second Xiangya Hospital, \\ Central South University, Changsha, Hunan 410011, P.R. China \\ Received February 27, 2015; Accepted March 10, 2016
}

DOI: $10.3892 / \mathrm{ol} .2016 .4432$

\begin{abstract}
The present study reports the case of a 42-year-old male with multiple myeloma (MM)-associated skin light chain amyloidosis who presented with skin purpura as the initial symptom, which was misdiagnosis as Henoch-Schönlein purpura nephritis prior to admission to the Second Xiangya Hospital (Changsha, Hunan, China). The patient presented with purpura, papules petechiae and spontaneous ecchymosis, which was located scattered around the neck, chest and limbs, accompanied by a small amount of bleeding in the conjunctival and oral mucosa, and a swollen tongue. Upon laboratory examination, the serum immunological change showed increased serum immunoglobulin $G$ and $\lambda$ light chain levels, and a urine Bence Jones protein level of $>1 \mathrm{~g} / 24 \mathrm{~h}$. This was accompanied with an abnormal result for immunofixation electrophoresis, and positive staining with Congo red showing apple-green birefringence in skin biopsy specimens. Thus, the patient was diagnosed with MM-associated skin amyloidosis with the initial symptom of skin purpura. Following treatment with chemotherapy consisting of prednisone and bortezomib, the skin lesions markedly improved. The present study indicates that the presentation of skin purpura in systemic amyloidosis associated with MM may be an important aid in the diagnosis and direct treatment of this disease in the clinic.
\end{abstract}

\section{Introduction}

Multiple myeloma (MM) is a clonal malignant disease of the plasma cells, characterized by the production of a monoclonal immunoglobulin, anemia, lytic bone lesions and renal failure (1). MM is divided into symptomatic, smouldering multiple myeloma and monoclonal gammopathy of

Correspondence to: Dr Lin Sun, Department of Nephrology, Second Xiangya Hospital, Central South University, 15th Science and Technology Building, 139 Renmin Road, Changsha, Hunan 410011, P.R. China

E-mail: sunlinnwu11@163.com

Key words: multiple myeloma, amyloidosis, skin purpura undetermined significance (2). Myeloma-associated amyloidosis is found in $12-15 \%$ of myeloma patients, among whom, $30 \%$ exhibit asymptomatic disease (3). The amyloid substance, which originates from immunoglobulin light chains (always of $\lambda$ type) produced by myeloma cells, is deposited in various tissues, leading to organ dysfunction, affecting the heart, liver, kidney, nervous system and gastrointestinal tract $(3,4)$. The diagnosis of MM-associated amyloidosis is confirmed based on positive amyloid tissue staining with Congo red and immunoglobulin light chain subtyping, evidence of a monoclonal plasma cell disorder and amyloid-related organ dysfunction (5). In addition, novel techniques are available for the early diagnosis, including immunofixation electrophoresis, bone marrow core biopsies with cluster of differentiation (CD)138 staining, and detection of the levels of circulating plasma cells, cytogenetic subtypes and abnormal plasma cell immunophenotype $(2,3)$. Recent novel agents, including lenalidomide, bortezomib and dexamethasone combination therapy, demonstrate favorable tolerability for this disease. It has been reported that MM-associated amyloidosis is an independent high-risk prognostic factor, while a similar 1-year survival rate $(\sim 80 \%)$ has been found in amyloid light-chain (AL) amyloidosis patients with or without concurrent myeloma $(1,3,6)$. MM-associated amyloidosis has complex and diverse clinical manifestations. Occasionally, the symptoms are not typical at the early stage of this disease, resulting in misdiagnosis. Cutaneous involvement, including macroglossia, purpura, petechiae and ecchymoses, are occasionally observed as elements among a constellation of signs, but rarely as the initial manifestation of systemic amyloidosis, particularly the myeloma-associated form $(5,7)$. The current study presents a case of skin purpura with mucosal bleeding and hematuria as the first symptom, which was later diagnosed as MM.

\section{Case report}

A 42-year-old male initially presented on December 15, 2013, with skin purpura and recurrent episodes of hematuria that had persisted for 6 months. The patient was diagnosed with Henoch-Schönlein purpura (HSP) nephritis at a local hospital and received prednisone (30 $\mathrm{mg}$ per day) for 3 months. Subsequent to treatment, the symptoms of purpura and hematuria persisted, so the patient was admitted to the Second Xiangya 

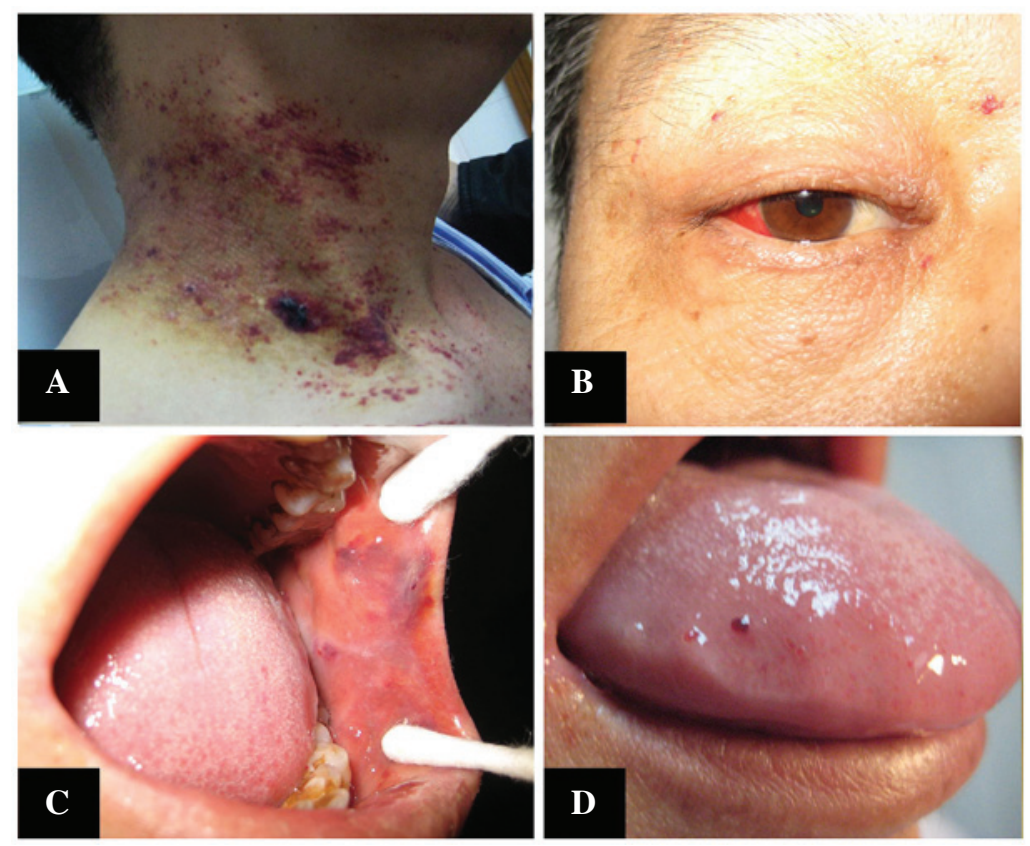

Figure 1. Distribution of skin purpura and the situation of the tongue. (A) Purpura distributed around neck. (B) Bulbar conjunctiva hemorrhage. (C) Petechia on the oral mucosa. (D) Petechia on the swollen tongue.
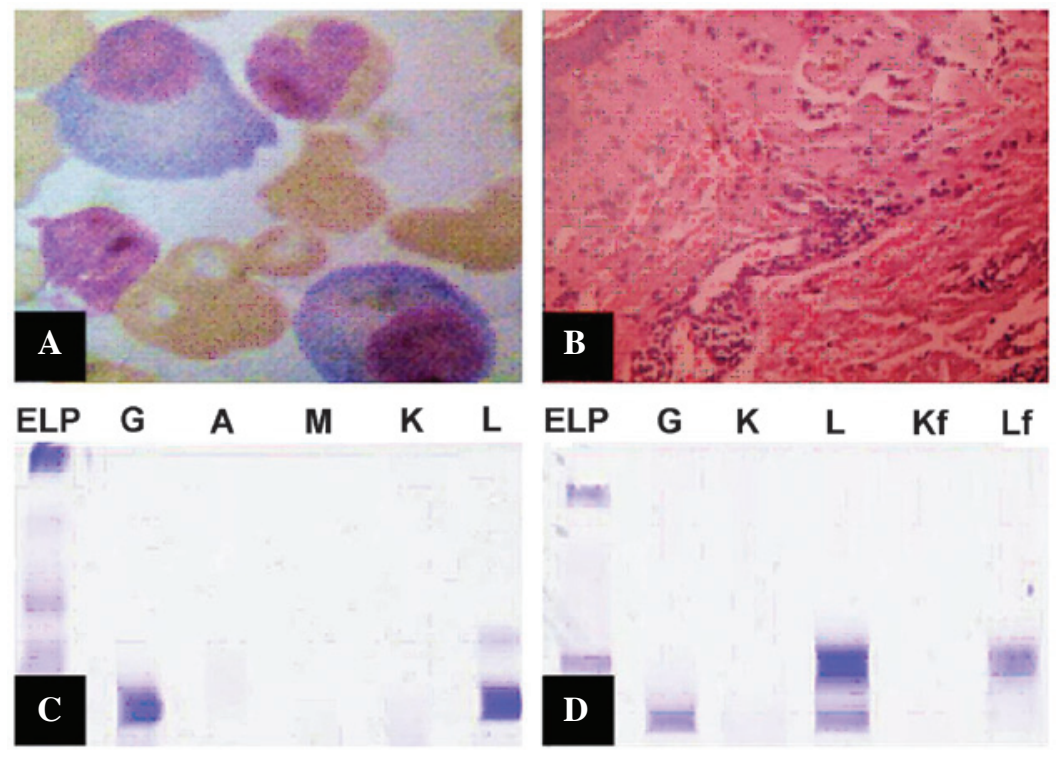

Figure 2. Laboratory test results. (A) Wright's-stained bone marrow aspirate smear showing the abnormal proliferation of plasma cells (x1,000 magnification), (B) Histopathological examination of the skin showing vascular endothelial cell proliferation and hemorrhage (hematoxylin and eosin stain; x200 magnification). Immunofixation electrophoresis of the (C) serum and (D) urine. ELP, positive immunofixation electrophoresis; Ig, immunoglobulin; G, IgG; A, IgA; M, IgM; K, $\kappa$ light chain; $L, \lambda$ light chain; Kf, free $\kappa$ light chain; Lf, free $\lambda$ light chain.

Hospital (Changsha, Hunan, China) on March 4, 2014, presenting with purpura, papules petechiae and spontaneous ecchymosis, which was located scattered around the neck, chest and limbs, accompanied by a small amount of bleeding in the conjunctival and oral mucosa, and a swollen tongue (Fig. 1), No itching, pain or oozing of the skin purpura was noted. In addition, the patient reported no dizziness, fatigue, bone pain, joint pain, hair loss, abdominal pain or fever. Blood tests to determine red blood cell (RBC), white blood cell, hemoglobin and platelet counts were normal, and renal function and the serum calcium level were also normal.
Urine analysis showed an abnormally high RBC count of $29,279.9 / \mu 1$ (normal range, $0-25 / \mu 1$ ) and a negative result for protein in the urine. The coagulation tests (prothrombin time, activated partial thromboplastin time and fibrinogen level) were within normal limits. The serum albumin level was $30.9 \mathrm{~g} / 1$ (reference range, 40-55 g/l), and tests for aspartate aminotransferase, alanine aminotransferase, total bilirubin and direct bilirubin for liver function were normal. Tests for human immunodeficiency virus, hepatitis B and hepatitis $\mathrm{C}$ were negative, but the erythrocyte sedimentation rate was $46 \mathrm{~mm} / \mathrm{h}$ (reference range, 0-15 mm/h). Serum 

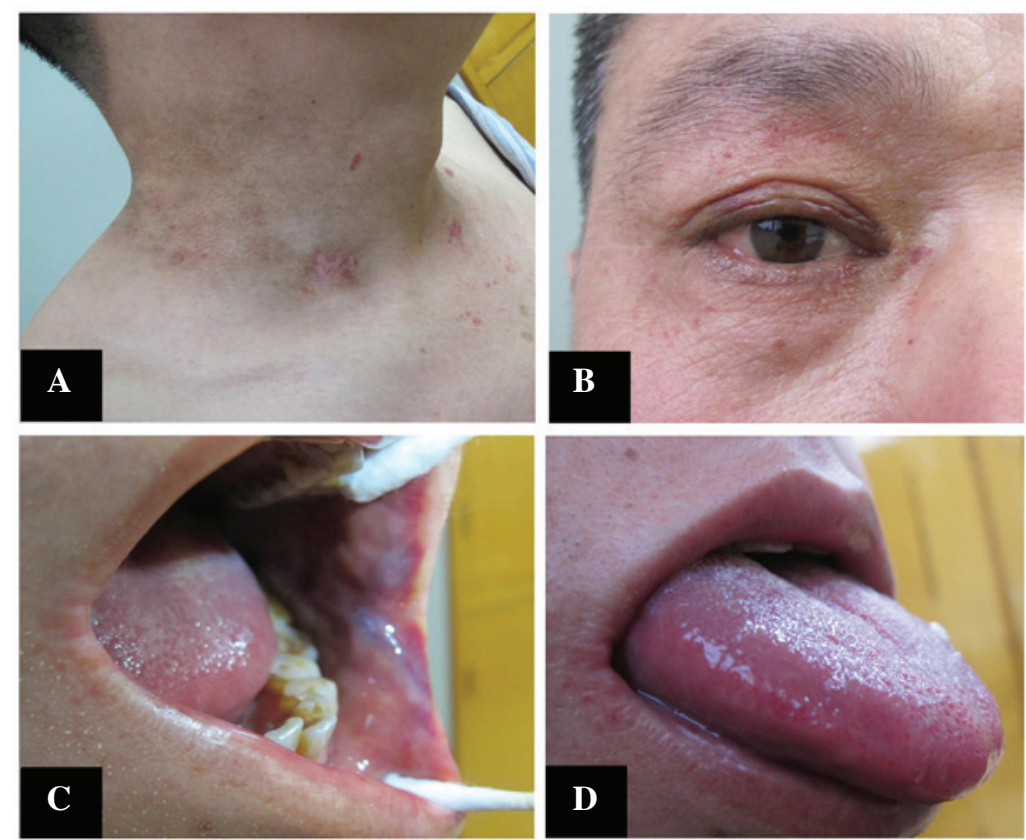

Figure 3. Changes visible on the (A) neck, (B) bulbar conjunctiva, (C) oral mucosa and (D) tongue following chemotherapy.

immunoglobulin (Ig) tests showed a high level of $\mathrm{IgG}$ at $21.60 \mathrm{~g} / 1$ (reference range, 7.23-16.8 g/l), but a decreased level of $\mathrm{IgA}$ at $0.24 \mathrm{~g} / 1$ (reference range, $0.69-3.82 \mathrm{~g} / \mathrm{l}$ ) and $\mathrm{IgM}$ at $0.16 \mathrm{~g} / 1$ (reference range, 0.63-2.77 g/l). C3 and C4 were normal, while the level of serum $\kappa$ light chain was $1.69 \mathrm{~g} / 1$ (reference range, 5.98-13.29 $\mathrm{g} / \mathrm{l}$ ) and the level of $\lambda$ light chain was $20.40 \mathrm{~g} / \mathrm{l}$ (reference range, 2.80-6.65 g/l); the ratio of $\kappa / \lambda$ was 0.08 (reference range, $1.47-2.95$ ), and urine light chain analysis revealed a type of Bence-Jones proteinuria with a monoclonal increase in $\lambda$ chain level ( $2.57 \mathrm{~g}$; reference range, $0-0.02 \mathrm{~g} / \mathrm{l})$ and a normal level of $\kappa$ chain. The $\kappa / \lambda$ ratio was 0.02 (reference range, $1.47-2.95$ ). In addition, serum protein electrophoresis was performed with the following results: $\beta 2$-microglobulin, $3.45 \mathrm{mg} / 1$ (reference range, $1-3 \mathrm{mg} / 1$ ); $\alpha 1$ microglobulin, $2.03 \%$ (reference range, 2.8-5.6\%); $\alpha 2$ microglobulin, 6.74\% (reference range, 8.4-14.2\%); and $\gamma$ globulin, $31.3 \%$ (reference range, 11.5-25.7\%). Other tests, including those for autoantibodies, such as anti-Sm anti-SSA, anti-SSB and anti-Jo-1 antibodies, were normal, as detected by an enzyme-linked immunosorbent assay kit (catalog no. 250402002-b; Changzhou Kelai Clinical Labratory, Changzhou, Jiangsu, China) following the manufacturer's protocols. Factor VIII activity was normal. Echocardiography and X-rays of the chest, skull and pelvis were normal; bone-marrow biopsy revealed increased plasma cells accounting for $16 \%$ of the marrow cellularity. Examination of skin biopsy specimens demonstrated vascular endothelial cell proliferation in the small blood vessels, hemorrhage and a large overflow of red blood cells. In addition, positive staining with Congo red showing apple-green birefringence was observed under polarized light microscopy (Fig. 2). Furthermore, positive immunohistochemistry showed a positive reaction for $\lambda$ light chain, and $\mathrm{T}$ cell markers (CD3, CD4, CD5, CD8 and CD43) were observed, as detected using rabbit polyclonal CD3 antibody (1:100 dilution; catalog no. ab5690), rabbit monoclonal CD4 antibody
(1:500 dilution; catalog no. ab133616), rabbit monoclonal CD5 antibody (1:250 dilution; catalog no. ab75877), rabbit polyclonal CD8 antibody with (1:200 dilution; catalog no. ab4055) and mouse monoclonal CD43 antibody (1:20 dilution; catalog no. ab89691) (all Abcam, Cambridge, UK), respectively. The specimens were negative for B cell marker proteins. Based on the history of plasmacytoma, the skin biopsy and the current presentation, a workup for MM [lipoarabinomannan (LAM)IgG merge-free light chain-type]-associated skin light chain amyloidosis was initiated. Since the MM was accompanied by amyloidosis, indicating the disease was active (1), the patient was referred to an oncologist who began treatment with a VDT regimen as follows: In the induction of remission therapy, $1.3 \mathrm{mg} / \mathrm{m}^{2}$ intravenous bortezomib on days $1,4,8$ and 11; $20 \mathrm{mg}$ daily intravenous dexamethasone on days 1, 2, 4, 5, $8,9,11$ and 12, every 4 weeks for 4 cycles; and $100 \mathrm{mg}$ thalidomide (daily). In addition, in post-remission therapy, $100 \mathrm{mg}$ thalidomide (daily) was prescribed for 1 year. Following six courses of chemotherapy, the skin lesions markedly improved (Fig. 3). In January 2015, follow-up examination showed that the serum IgG level was 17.82 and the serum $\lambda$ light chain level was $8.82 \mathrm{~g} / 1$, which were almost normal levels.

\section{Discussion}

In the present case, the patient presented with skin purpura and hematuria, without proteinuria or clear bone destruction and anemia, which can be misdiagnosed as HSP nephritis. However, following treatment with prednisone for 3 months, the purpura and hematuria did not improve. HSP is a small-vessel vasculitis that affects the skin, joints, gut and kidneys. The feature of a rash in HSP presents as palpable purpuric vasculitis on the lower limbs spreading over the extensor surfaces to the buttocks and occasionally to the upper limbs, while skin lesions often occur on the lips, periorbital regions and eyelids of MM patients, accompanied 
with macroglossia (8-10). Thus, the location of the purpura and skin biopsy is critical in differentiating between the diagnosis of MM-associated AL-amyloidosis and HSP. It is believed that skin purpura and lesions can also appear in autoimmune diseases and tumors, particularly in malignancies of the blood, such as MM. Therefore, further tests for serum immunological changes were performed in the present study, together with a skin biopsy. Increased serum IgG levels, as detected by serum Ig tests, increased $\lambda$ chain levels, as detected by serum and urine light chain analysis, and a urine Bence Jones protein level of $>1 \mathrm{~g} / 24 \mathrm{~h}$ was accompanied by an abnormal result for immunofixation electrophoresis, and positive staining with Congo red showing apple-green birefringence in the skin biopsy specimens. Thus the patient was diagnosed with MM-associated skin amyloidosis with the initial symptom of skin purpura.

AL amyloidosis is almost always associated with a plasma cell dyscrasia. In total, $20 \%$ of amyloidosis is caused by MM, however, only $5-15 \%$ of patients with MM develop amyloid light chain deposits (11). A previous study reported a case of MM preceded by vascular purpura with cutaneous leukocytoclastic viscosity (12). It has also been reported that the purpura, papules, spontaneous ecchymosis and petechiae are occasionally the first and only sign of systemic amyloidosis (13). However, there have been no studies reporting MM (LAM-IgG light chain-type)-associated skin amyloidosis with skin purpura as the initial symptom. The present study discussed a case of MM-associated amyloidosis, in which the patient first presented with skin purpura and hematuria, without other systemic damage, and was thus misdiagnosed with purpura nephritis in the early stage. Furthermore, MM-associated AL amyloidosis also varies from other types of kidney disease, including chronic nephritis, where patients present with hematuria, proteinuria and anemia, which are usually observed in advanced stages of chronic nephritis. However, hypertension can also often be observed in chronic nephritis with impaired renal function. The mechanisms that cause intracutaneous bleeding may include abnormal $\mathrm{M}$ protein (14) and injury by tumorocellular infiltration or amyloidosis to the fragile vascular wall (15). Of course, these presentations of skin purpura are not specific to systemic amyloidosis associated with MM, but they may be an important aid to the diagnosis and direct treatment of this disease in the clinic.

\section{References}

1. Vela-Ojeda J, García-Ruiz Esparza MA, Padilla-González Y, Sánchez-Cortes E, García-Chávez J, Montiel-Cervantes L, Reyes-Maldonado E, Majluf-Cruz A and Mayani H: Multiple myeloma-associated amyloidosis is an independent high-risk prognostic factor. Ann Hematol 88: 59-66, 2009.

2. Rajkumar SV, Dimopoulos MA, Palumbo A, Blade J, Merlini G, Mateos MV, Kumar S, Hillengass J, Kastritis E, Richardson P, et al: International Myeloma Working Group updated criteria for the diagnosis of multiple myeloma. Lancet Oncol 15: e538-e548, 2014.

3. Dinner S, Witteles W, Witteles R, Lam A, Arai S, Lafayette R, George TI, Schrier SL and Liedtke M: The prognostic value of diagnosing concurrent multiple myeloma in immunoglobulin light chain amyloidosis. Br J Haematol 161: 367-372, 2013.

4. Girnius S, Seldin DC, Skinner M, Finn KT, Quillen K, Doros G and Sanchorawala V: Short and long-term outcome of treatment with high-dose melphalan and stem cell transplantation for multiple myeloma-associated AL amyloidosis. Ann Hematol 89: 579-584, 2010

5. Ohashi T, Kikuchi $\mathrm{N}$ and Yamamoto $\mathrm{T}$ : Unusual milia amyloidosis as initial signs of multiple myeloma-associated systemic amyloidosis. Int J Dermatol 52: 981-982, 2013.

6. Nagano S, Mori M, Kato A, Ono Y, Aoki K, Arima H, Takiuchi Y, Tabata S, Yanagita S, Matsushita A, et al: Therapeutic effects of lenalidomide on hemorrhagic intestinal myeloma-associated AL amyloidosis.Intern Med 52: 1101-1105, 2013.

7. Xu J, Tahan S, Jan F, Do D and Wu H: Nail dystrophy as the initial sign of multiple myeloma-associated systemic amyloidosis. J Cutan Pathol: Mar 8, 2016 (Epub ahead of print).

8. Vella FS, Simone B and Antonaci S: Palmodigital purpura as the only skin abnormality in myeloma-associated systemic amyloidosis.Br J Haematol 120: 917, 2003.

9. Oliver AJ: Multiple myeloma presenting with amyloid purpura and macroglossia: A case report and literature review. Compendium 15: 712, 714-716, 1994.

10. Yücel A, Akman A, Denli YG, Acar MA, Karakas M, Hazar B, Gümürdülü D, Ergin M, Memisoglu HR: A case of systemic amyloidosis associated with multiple myeloma presented as macroglossia and purpura. J Eur Acad Dermatol Venereol 18: 378-379, 2004

11. Lee HJ, Chang SE, Lee MW, Choi JH and Moon KC: Systemic amyloidosis associated with multiple myeloma presenting as periorbital purpura. J Dermatol 35: 371-372, 2008.

12. Peterlin P, Ponge T, Blin N, Moreau P, Hamidou M and Agard C: Paraneoplastic cutaneous leukocytoclastic vasculitis disclosing multiple myeloma: A case report. Clin Lymphoma Myeloma Leuk 11: 373-374, 2011.

13. Tuñón J, Oliva-Encabo R and Cortés M: Diagnosis of cardiac amyloidosis by skin lesions. Rev Esp Cardiol 67: 666, 2014.

14. Eby CS: Bleeding and thrombosis risk in plasma cell dyscrasias. Hematology Am Soc Hematol Educ Program 2007: 158-164, 2007.

15. Lipsker D and Boeckler P: Cutaneous manifestations of paraproteinemia and their mechanisms. Presse Med 36: 1135-1140, 2007 (In French). 